\title{
Rectification of chaotic fluid motion in two-dimensional turbulence
}

\author{
N. Francois, ${ }^{*}$ H. Xia, H. Punzmann, and M. Shats \\ Research School of Physics and Engineering, Australian National University, Canberra, \\ Australian Capital Territory 2601, Australia
}

(Received 10 June 2018; published 4 December 2018)

\begin{abstract}
Turbulence is a mechanism leading to energy dissipation, however it also accumulates energy by spreading it over a range of scales. This valuable energy reservoir is known as the inertial interval. The broader this interval is, the more energy is stored and an interesting question is whether it is possible to efficiently use this energy. Recent advances in the understanding of turbulence rely on the trajectory-based or Lagrangian description of the flow. Here we show how to extract energy from the inertial interval of two-dimensional turbulence by taking advantage of its fine Lagrangian structure. A floating object in wavedriven turbulence can exploit the fluid erratic motion to fuel either directional propulsion or rotation. The shape of the object controls its ability to become a vehicle or a rotor that can tap the energy of correlated bundles of fluid trajectories. These findings offer methods of creating self-propelled devices or turbines utilizing the energy of turbulence.
\end{abstract}

DOI: 10.1103/PhysRevFluids.3.124602

\section{INTRODUCTION}

Turbulence is a common paradigm of unpredictable and chaotic flows [1,2]. In both threedimensional (3D) and 2D turbulence, nonlinear interactions spread energy over the inertial interval. Through this process a substantial amount of energy is stored into turbulent fluctuations. Current technologies commonly used to collect renewable energy cannot operate properly in strongly turbulent environment [3]. Developing methods to collect energy stored in turbulent fluctuations requires advanced knowledge of the structure of the turbulent flow. Due to progress in experimental methods, it is now possible to describe turbulent flow in the Lagrangian frame, the reference frame of fluid particles advected by the flow. The Lagrangian description of turbulence has recently advanced our knowledge significantly in many enduring problems, for instance, in fundamental questions related to statistical properties of homogeneous and isotropic turbulence but also in many applications based on mixing or transport in turbulent flows $[4,5]$.

There is a growing realization that $2 \mathrm{D}$ turbulence is far more ubiquitous than it was originally expected [6-10]. Two-dimensional turbulence supports an inverse energy cascade that transfers energy from small to larger scales. When bounded, such flows can self-organize to form large-scale coherent flows in a process known as spectral condensation [11-14]. The remarkable ability of $2 \mathrm{D}$ turbulence to self-organize in the presence of boundaries promises versatile methods of energy conversion. It has recently been found that disordered surface waves generate horizontal turbulent motion at the water surface $[14,15]$. Despite the 3D nature of the fluid motion in the waves, the statistics of horizontal velocity fluctuations is consistent with 2D turbulence $[10,16]$. In particular, there is an inertial transfer of kinetic energy from smaller to larger scales [11]. In this system, both Eulerian (field representation) and Lagrangian (trajectory-based) features of the chaotic flows can be accurately measured over a broad range of kinetic energies [16-18].

*nicolas.francois@anu.edu.au 
Here we show that the underlying Lagrangian structure of wave-driven 2D turbulence allows the rectification of the fluid velocity fluctuations to create either a stationary rotor or a floating vehicle capable of propelling along the turbulent liquid-gas interface. We demonstrate experimentally the operational principle of these self-propelled vehicles or rotors by studying the coupling of the device geometry with the Lagrangian fabric of 2D turbulence. These devices exploit fluctuations of finiteReynolds-number flows possessing structural and temporal memory.

\section{EXPERIMENTAL RESULTS}

Turbulent flows in these experiments are generated on the water surface in vertically shaken containers $[14,15]$. Above a critical vertical acceleration, parametrically excited waves, or Faraday waves, appear at the liquid-gas interface. When the Faraday waves are steep, the wave motion is disordered [14]. We study these waves in the range of vertical accelerations $a$ which corresponds to the supercriticality factor of $0.2<\epsilon<2$, where $\epsilon=\left(a-a_{\mathrm{th}}\right) / a_{\mathrm{th}}$ and $a_{\mathrm{th}}$ is the instability threshold of the Faraday waves (see the Supplemental Material [19]). Though energy is injected into vertical motion of a liquid, it is converted into chaotic horizontal fluid motion via the generation of horizontal surface vortices [16,20,21]. These Faraday wave-driven flows are random and show no component of mean flow. The kinetic energy of the horizontal flow $E_{f}$ is proportional to the square of the horizontal velocity fluctuations, $E_{f} \sim U^{2}$ ( $U$ is the rms value of the fluctuations). The horizontal flow energy increases with the amplitude of the vertical acceleration as $E_{f} \sim \epsilon^{2}$. The flow is forced at the scale $L_{f} \approx \lambda / 2$, where $\lambda$ is the wavelength [14,16,17]. Figure 1(a) illustrates the chaotic transport of tracer particles at the surface perturbed by Faraday waves. Transport properties of these flows have been studied previously $[10,15,16,22-24]$. It has been shown that the single-particle dispersion has a diffusive nature. In other words, a fluid particle on the surface executes a random walk and its mean-square displacement from its original position increases linearly with time [17] [Fig. 1(b)]. Moreover, the velocity fluctuations of such flows were found to follow a Gaussian distribution, as demonstrated in Fig. 1(c). The rms velocity $U$ depends on the kinetic energy accumulated over a broad range of scales, the inertial range, by a $2 \mathrm{D}$ process referred to as the inverse energy cascade $[11,14,15]$. The latter corresponds to a transfer of energy from the injection scale $L_{f}$ towards larger scales. It is identified by a $k^{-5 / 3}$ scaling in the wave-number spectrum of the horizontal kinetic energy [see Fig. 1(d)]. In these experiments, the inverse cascade spreads energy up to scales close to $10 L_{f}$. This upper bound also determines the size of the largest domain in which spectral condensation occurs experimentally [14].

\section{A. Self-propulsion versus drift motion of floating objects on the water surface}

Figure 1(a) shows the trajectory (in red) of a floating disk placed into the wave-driven flow [see schematics in Fig. 1(e) and the Supplemental Material [19] for a description of the material, density, and thickness of the floaters used here]. The floater is much larger than the characteristic flow scale $L_{f}$ and its motion is erratic. The motion of large floating disks is diffusive at long times, as seen in Fig. 1(b). The diffusion coefficient of the disk floater is 10 times lower than that measured using fluid tracers. It has been recently demonstrated that for large circular objects (size much greater than $\lambda$ ) drifting in the Faraday waves there exists a relation between the wave-driven diffusion and the drag coefficient [25], analogous to the fluctuation-dissipation theorem used in equilibrium statistical mechanics. However, there is ample evidence that wave-driven flows show many properties of twodimensional turbulence, a strongly out-of-equilibrium state [14-16].

The underlying nonequilibrium nature of the flow can be revealed by changing the shape of the large floater. Instead of a simple disk, we use a bracket-shaped floater (a floater with two L-shaped arms). Figure 1(f) shows the trajectory of this winged vehicle placed into the flow. The vehicle can propel itself along a smooth line over long distance.

To understand the mechanism of this propulsion, we study the motion of floaters which rotate freely about the $z$ axis with a fixed pivot point in the horizontal plane. We thus turn winged vehicles 


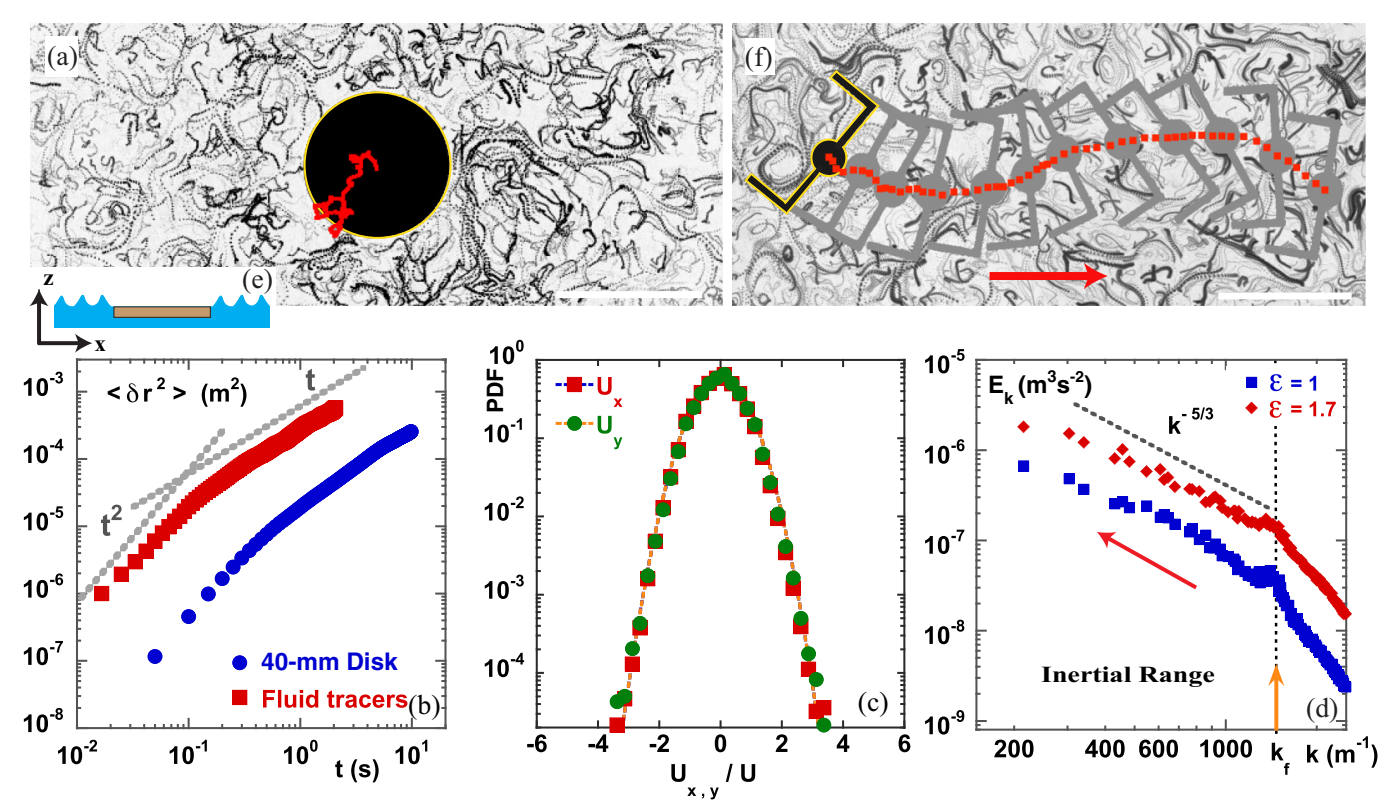

FIG. 1. Disk floater and self-propelled vehicle in turbulent flows. (a) Fluid particle streaks around a 40-mm-diam floating disk. The trajectory of the disk floater (red dots) in the turbulent flow (the parameters are $U=3.7 \times 10^{-2} \mathrm{~m} \mathrm{~s}^{-1}$ and $L_{f}=4.4 \mathrm{~mm}$ ) is shown with a scale bar equal to $40 \mathrm{~mm}$. The floater motion is tracked for $15 \mathrm{~s}$; for clarity, particle streaks are only $0.7 \mathrm{~s}$ long. (b) Mean-square displacement of fluid particles and of the large disk floater. (c) Probability distribution function (PDF) of the components $U_{x}$ and $U_{y}$ of the flow velocity along the $x$ and $y$ axes. The orange and blue dashed lines are Gaussian fits. (d) Wave-number spectrum $E_{k}(k)$ of the horizontal kinetic energy of the wave driven flow for two different supercriticality factors $\epsilon$. The red arrow indicates the direction of the energy flux and the orange arrow indicates the forcing wave number $k_{f}=2 \pi / L_{f}$. (e) Schematic of the large neutrally buoyant object at the water surface. (f) Trajectory of the winged vehicle (red dots) in the turbulent flow (the wing span of the vehicle is $40 \mathrm{~mm}$ and the red arrow indicates the direction of motion). The vehicle motion is tracked for $24 \mathrm{~s}$; for clarity, particle streaks are only $0.5 \mathrm{~s}$ long (the scale bar is equal to $40 \mathrm{~mm}$ ).

into rotors. We study two types of rotors: a beam rotor [Fig. 2(a)] and a chiral Z rotor which is made of two L-shaped arms [Fig. 2(b)]. The rotation axes are at the rotor geometric center. When the beam is placed on the fluid surface [Fig. 2(a)] its orientation or its polar angle $\theta(t)$ fluctuates about its initial value and the time-integrated angular displacement $S_{\theta}=\int \dot{\theta}(t) d t$ remains close to zero. The motion of the $Z$ rotor [Fig. 2(b)] fluctuates but it also steadily rotates in one direction (see movie 1 in [19]). The rotation is always such as if a force is exerted from the inside of the L-shaped arm [clockwise rotation in Fig. 2(b)]. The angular displacement $S_{\theta}$ increases linearly with time for the $\mathrm{Z}$ rotor [Fig. 2(c)]. Its average angular velocity $\left\langle V_{\theta}\right\rangle=\langle\dot{\theta}(t)\rangle$ is therefore steady in time and it increases with the increase in the turbulent velocity fluctuations $U$ [Fig. 2(d)]. The rotation direction reverses when the $\mathbf{Z}$ rotor is placed upside down.

The square of the angular velocity $\left\langle V_{\theta}\right\rangle^{2}$ is linearly proportional to the kinetic energy stored in the fluctuations of the horizontal flow velocity. This is observed in a broad range of the turbulent flow energies $E_{f} \sim U^{2}$, over two orders of magnitude as seen in Fig. 2(e). The probability distribution function of the angular velocity fluctuations $V_{\theta}-\left\langle V_{\theta}\right\rangle$ is Gaussian with a distribution width comparable to the mean angular velocity $\left\langle V_{\theta}\right\rangle$ [see the inset in Fig. 2(e)]. This indicates a stochastic propulsive mechanism. The energy transfer from the turbulent flow to the $Z$ rotor also depends on both the rotor size and the flow characteristic scale $L_{f}$. Figure 2(f) shows the rotational energy $J\left\langle V_{\theta}\right\rangle^{2}$ as a function of the normalized scale $L_{r} / L_{f}$, where $J$ is the moment of inertia of the 

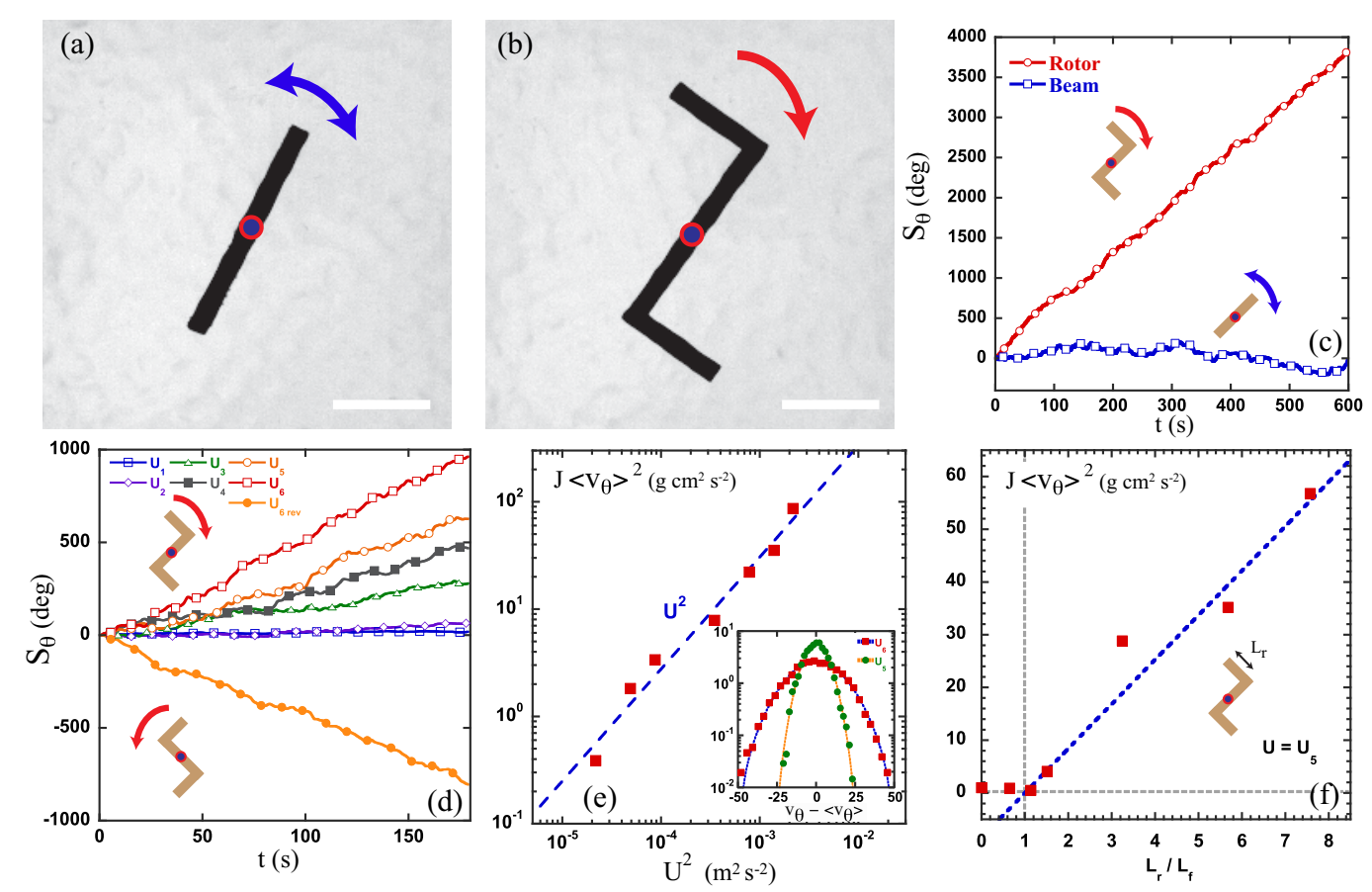

FIG. 2. Rotors. (a) and (b) A single beam and a $Z$ rotor in a 2D turbulent flow. The two structures can rotate freely about the $z$ axis but are fixed in the $x-y$ plane (the blue dot marks the axis of rotation and the scale bar is equal to $20 \mathrm{~mm}$ ). (c) Cumulative angular displacement $S_{\theta}=\int \dot{\theta}(t) d t$ versus time. The beam rotates erratically with a mean angular position $\langle\theta(t)\rangle$ close to zero while the random rotation of the $\mathbf{Z}$ rotor is biased along a direction prescribed by its chirality. (d) Angular displacement $S_{\theta}$ versus time measured for increasing rms flow velocity $U$ and when the rotor handedness is reversed [rms velocity range $U=10^{-3}-\left(5 \times 10^{-2}\right) \mathrm{m} \mathrm{s}^{-1}$ ]. (e) Angular kinetic energy $J\left\langle V_{\theta}\right\rangle^{2}$ of the rotor versus mean kinetic energy $U^{2}$ of the flow (the dashed blue line is a linear fit; the parameters are $L_{f}=4.4 \mathrm{~mm}, L_{r}=25 \mathrm{~mm}$, and $J=3.1 \mathrm{~g} \mathrm{~cm}^{2}$ ). The inset shows the PDF of the fluctuations $V_{\theta}-\left\langle V_{\theta}\right\rangle$ of the $Z$ rotor measured in flows with different mean kinetic energies $U^{2}$ and forcing scale $L_{f}$ [the parameters are $\left(U_{5}=3.7 \times 10^{-2} \mathrm{~m} \mathrm{~s}^{-1}, L_{f}=3 \mathrm{~mm}\right)$ and $\left(U_{6}=4.7 \times 10^{-2} \mathrm{~m} \mathrm{~s}^{-1}, L_{f}=\right.$ $4.4 \mathrm{~mm}$ )]. (f) Angular kinetic energy $J\left\langle V_{\theta}\right\rangle^{2}$ versus the ratio $L_{r} / L_{f}$ at fixed $U$ [the dashed blue line is a linear fit; the parameters are $L_{f}=3-8 \mathrm{~mm}, L_{r}=5-25 \mathrm{~mm}$, and $\left.J=\left(1.9 \times 10^{-2}\right)-3.1 \mathrm{~g} \mathrm{~cm}^{2}\right]$.

rotor and $L_{r}$ is the size of the L-shaped arms [see the schematic in Fig. 2(f); by definition $L_{r}=0$ for a single blade]. The rectification of the fluctuations occurs above a threshold value of $L_{r} / L_{f}$. No unidirectional rotation is observed for small rotors $L_{r}<L_{f}$. For larger rotors, the rotational energy $J\left\langle V_{\theta}\right\rangle^{2}$ is an increasing function of the normalized size $L_{r} / L_{f}$.

\section{B. Fine structure of the turbulent flow}

The rotor size dependence in Fig. 2(f) suggests the presence of coherent structures coexisting with the chaotic flow background. To confirm the existence of and quantify statistically this persistent fabric we compute two types of Lagrangian correlation functions: a single-particle velocity autocorrelation $\rho_{11}(\tau)=\left\langle\mathbf{u}\left(t_{0}+\tau\right) \mathbf{u}\left(t_{0}\right)\right\rangle / U^{2}$ and a cross-correlation function $\rho_{12}\left(\tau, d_{12}\right)=$ $\left\langle\mathbf{u}_{1}(\tau) \mathbf{u}_{2}(\tau)\right\rangle / U^{2}$, where $\langle\cdot\rangle$ denotes the statistical averaging and $d_{12}$ is the initial $(\tau=0)$ distance between particles in pairs. These functions are referred to as Lagrangian because they are computed on horizontal velocities $\mathbf{u}$ measured in time along the trajectories of fluid tracers. The time integrals 

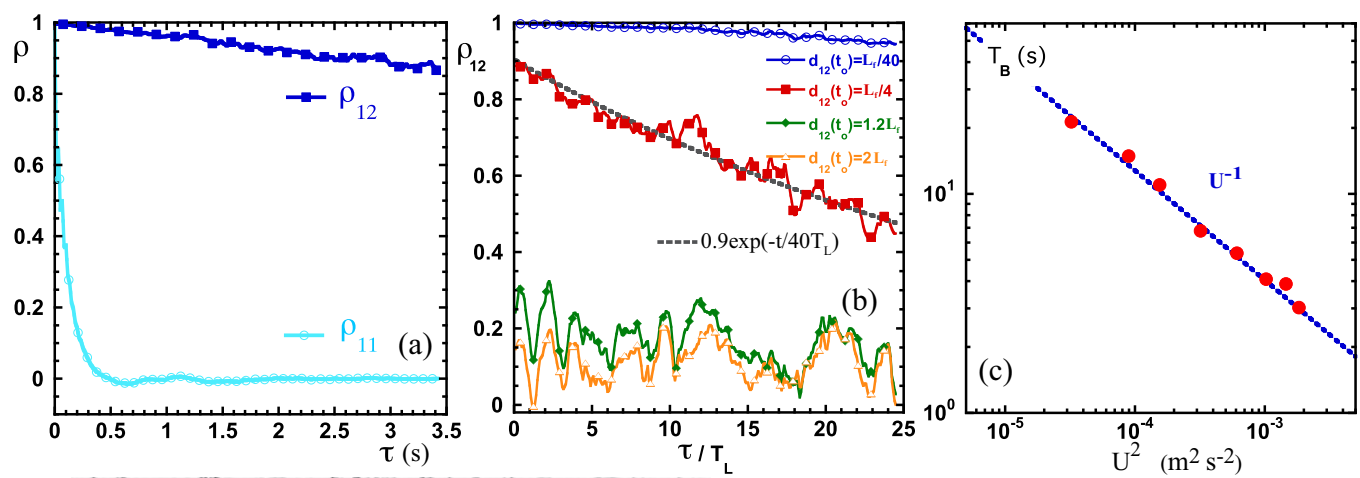

(d)

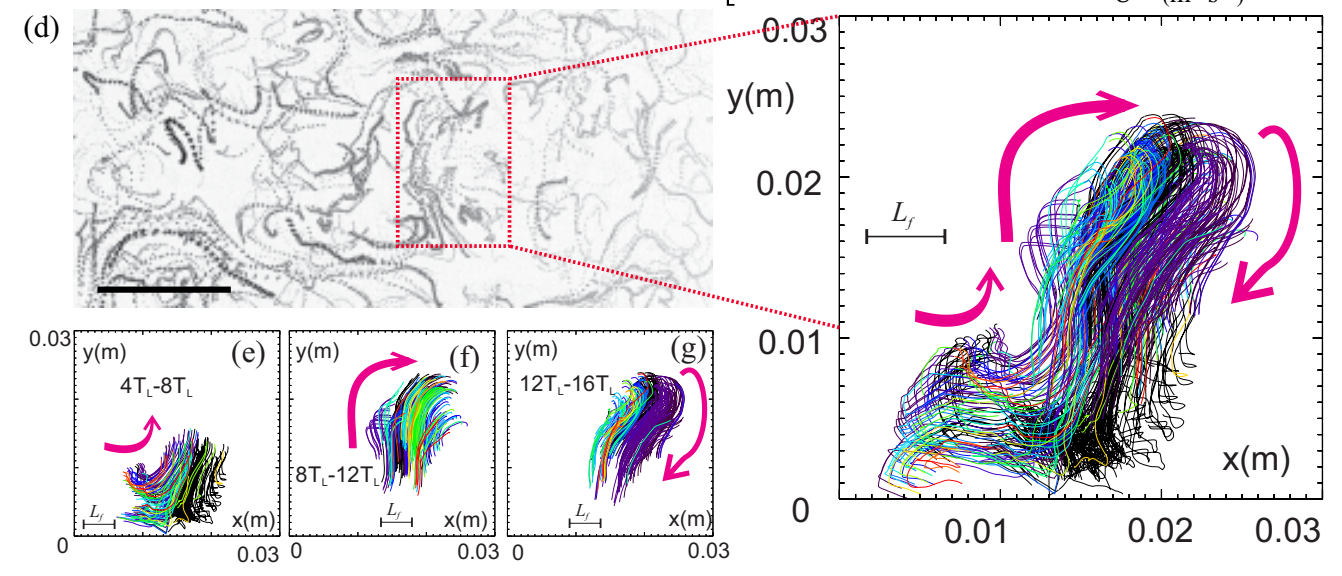

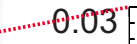
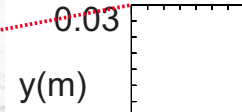

\begin{abstract}
$1+1+1$
\end{abstract}
$\left(\mathrm{m}^{2} \mathrm{~s}^{-2}\right)$

FIG. 3. Trajectories bundles: the fine fabric of turbulent flows. (a) Lagrangian autocorrelation $\rho_{11}$ (single particle) and cross-correlation $\rho_{12}$ (two particles) functions versus time. (b) Cross correlation $\rho_{12}$ versus time for increasing initial distance $d_{12}(\tau=0)$ between the two particles. Time is expressed in $T_{L}$ units $\left(T_{L}\right.$ is the single-particle autocorrelation time) and $d_{12}$ in $L_{f}$ units. (c) Bundle correlation time $T_{B}$ versus the flow mean kinetic energy $U^{2} ; T_{B}$ is computed by integration of $\rho_{12}$ over time for a distance $d_{12}=L_{f} / 4$. The measurements give the relation $T_{B} \approx 40 T_{L}$. (d) Fluid particle streaks in a turbulent flow and close-up of a trajectory bundle detected via the braid analysis (the parameters are 0.7 -s-long streaks, $L_{f}=4.4 \mathrm{~mm}$, and $U=$ $3.7 \times 10^{-2} \mathrm{~m} \mathrm{~s}^{-1}$; the scale bar is equal to $20 \mathrm{~mm}$ ). Details on the temporal evolution of the bundle shown in (d) are shown at different time intervals: (e) $4 T_{L}-8 T_{L}$, (f) $8 T_{L}-12 T_{L}$, (g) $12 T_{L}-16 T_{L}$ (scale bars show the forcing scale $L_{f}$ ).

of the functions $\rho_{11}(\tau)$ and $\rho_{12}(\tau)$ give two Lagrangian timescales $T_{L}$ and $T_{B}$, respectively, which characterize the memory of the flow.

A single particle forgets about its initial direction on the timescale of $T_{L}$. The vector velocities of two particles which are initially close to each other $\left(d_{12} \ll L_{f}\right)$ stay correlated for much longer time, $T_{B} \approx 250 T_{L}$ in Fig. 3(a). If the initial particle separation $d_{12}$ is gradually increased, the pair correlation time $T_{B}$ decreases [Fig. 3(b)]. The cross-correlation function $\rho_{12}$ decays exponentially with time when $d_{12}<L_{f}$. At large separations $d_{12}>L_{f}$, an abrupt transition occurs: $\rho_{12}$ collapses to a low level $\left(\rho_{12}<0.25\right)$. This suggests that a large number of fluid particles travel together along riverlike structures whose widths are about $L_{f}$. These bundles of trajectories stay together for a long time without separating. Indeed, the average lifetime of these structures is typically $\left\langle T_{B}\right\rangle_{d_{12}} \approx 30 T_{L}$ [when $T_{B}\left(d_{12}\right)$ is averaged over $d_{12}<L_{f}$ ]. The correlation time of a bundle $T_{B}$ decreases with the increase in the flow energy: $T_{B}$ is inversely proportional to the rms fluctuation velocity $U$ [Fig. 3(c)]. 
Visualizing the presence of these bundles directly from experimental trajectories is challenging because the temporal traces of the fluid particles in turbulent flows often appear as erratically entangled strands [see Fig. 3(d)]. Here we employ a recently developed method based on the concept of topological braids to detect coherent features in chaotic flows [26] (see the Supplemental Material [19]). This method reveals that locally many trajectories closely follow each other for rather long periods of time [Figs. 3(d)-3(g)]. Each of the bundles experiences occasional splitting into two, yet most strands stay together untangled and execute a complex collective motion. These topological bundles have a width close to $L_{f}$. Their average lengths, however, are substantially longer, as reflected by their long lifetime $\left\langle T_{B}\right\rangle_{d_{12}} \approx 30 T_{L}$ [Fig. 3(b)].

As shown in Fig. 3(a), the timescale $T_{L}$, which characterizes the single-particle dispersion in the turbulent flows, is much shorter than $T_{B}$. Recently it was shown that the fluid tracers have a diffusive behavior at long times $\left(t>T_{L}\right)$ with a turbulent diffusion coefficient $D_{t} \approx U L_{f}$ (17). Therefore, a single fluid particle executes a random walk whose characteristic step size and timescale are $L_{f}$ and $T_{L}$, respectively, with $U T_{L} \approx L_{f}$. Figures 3(d)-3(g) illustrate how the random-walk behavior, which dominates the single-particle statistics, coexists with correlated bundles: The random walk is executed by the bundles, rather than by individual uncorrelated trajectories.

\section{Coupling of the flow to the rotor}

Coherent bundles are constantly and randomly bent by the surrounding turbulent flow [18] [Fig. 3(d)]. When a winged vehicle, or a rotor, is introduced into the flow, coherent bundles are guided in the vicinity of the floater by their boundaries. To visualize this guiding effect, we fix the rotor to prevent its rotation and perform particle image velocimetry measurements near its corner, as shown in Fig. 4(a). By summing up instantaneous velocity fields over time intervals of several $T_{L}$, we can average out chaotic velocity fluctuations while retaining coherent features with longer lifetime. In Fig. 4(b) the time-averaged velocity field reveals bundles of trajectories that are guided and bent by the corner of a rotor. Inside the $L$ bend, the bundle of trajectories does not close onto itself to form a steady vortex structure as could have been expected; instead it is deformed along a curve whose curvature radius is similar to the bundle characteristic width $L_{f}$.

Given that wave-driven flows are dominated by inertial effects, a simplified picture emerges here as follows. The corner of the rotor acts as a stagnation point for the flow which forces the bundles to bend, thus creating a reaction force $\mathbf{F}_{\mathbf{p}}$ acting on the rotor's corner (in response to the centripetal acceleration experienced by the bundle), as illustrated in Fig. 4(c). Though the guided motion of the fluid bundles can be either clockwise or counterclockwise, the direction of the reaction force $\mathbf{F}_{\mathbf{p}}$ stays the same and points along the bisector of the corner. This force generates a propulsive torque $P$ acting on the rotor when the rotation axis is in its center. To test this hypothesis, we performed experiments using a half rotor. The pivot point of this corner-shaped rotor can be placed either at its vertex [Fig. 4(d)] or at the end of one of its arms [Fig. 4(e)]. The comparison between these two configurations shows that only the rotor whose vertex is free rotates. This confirms that the main component of the force moving the rotor acts at its corner. In the case of a winged vehicle [Fig. 1(f)], the forces $\mathbf{F}_{\mathbf{p}}$ acting on the symmetrically placed arms generate a directional propulsion.

\section{DISCUSSION}

Our results uncover a mechanism of exploiting the energy accumulated in the inertial range of 2D turbulent flows. The Lagrangian nature of this mechanism allows us to explain both the locomotion and the rotation at the fluid surface.

Since our stochastic rotor reaches a statistical steady state, that is, $\left\langle V_{\theta}\right\rangle$ is constant in time [Fig. 2(d)], we propose a simple model to describe its average motion. In this model, the mean torque $P$ exerted by the flow on the rotor is balanced by a linear dissipative torque $P=C_{s}\left\langle V_{\theta}\right\rangle$, where $C_{s}$ is a drag coefficient. The propulsive torque is modeled by $P=L_{R}\left(U^{2} / L_{f}\right) f\left(T_{B}\right)$, where $U^{2} / L_{f}$ is the centripetal acceleration characterizing the bending of coherent bundle with the rms velocity 


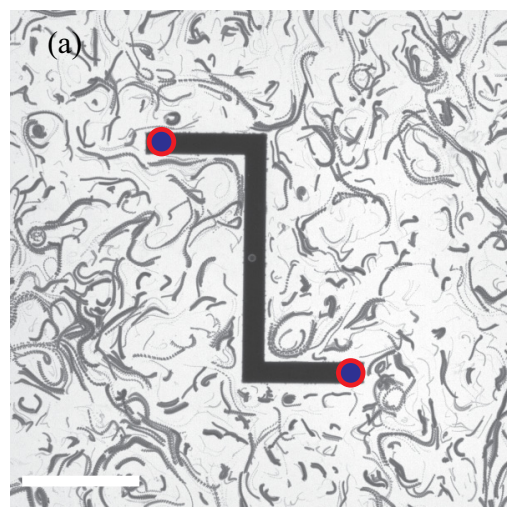

(c)

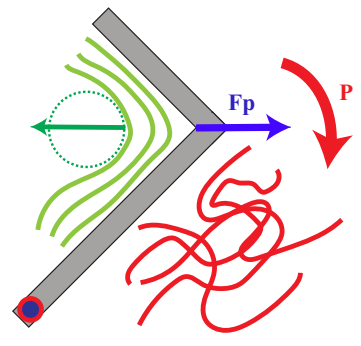

(d)

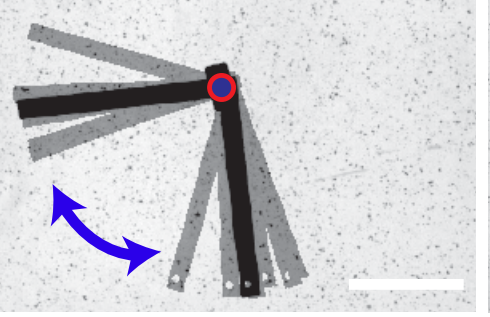

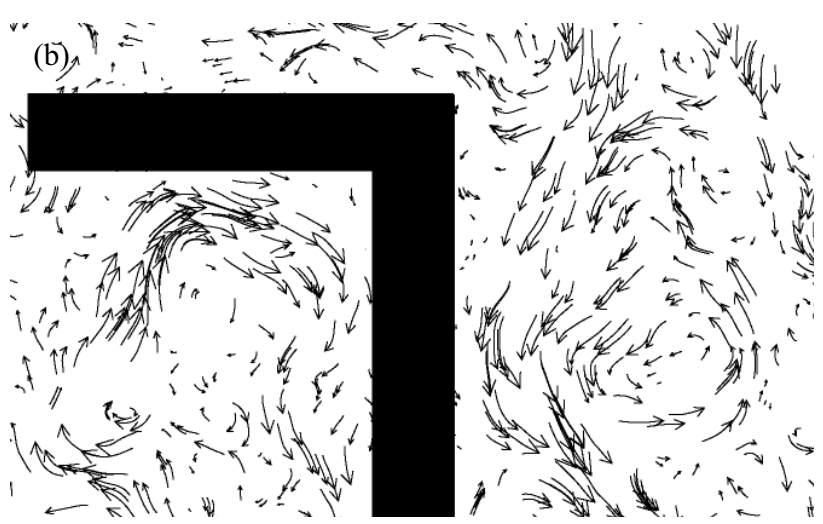

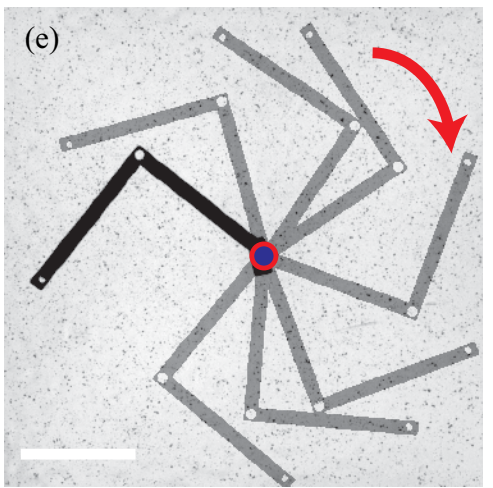

FIG. 4. Flow-rotor coupling. (a) Fluid particle streaks around a fixed $Z$ rotor. The rotor rotation is prevented by two anchoring axes (blue dots) (the parameters are streaks recorded over $0.66 \mathrm{~s}, L_{f}=4.4 \mathrm{~mm}$, and $U=9.3 \times 10^{-3} \mathrm{~m} \mathrm{~s}^{-1}$; the scale bar is equal to $25 \mathrm{~mm}$ ). (b) Velocity field measured using PIV in the corner of a fixed rotor $\left(T_{L} \approx 0.2 \mathrm{~s}\right.$, the velocity field is averaged over $1 \mathrm{~s}$, and the arrow length is proportional to the velocity magnitude). (c) Schematic of the modeling of the torque $P$ exerted by the fluid on the rotor. Here $\mathbf{F}_{\mathbf{p}}$ is the force resulting from the bending of a fluid bundle (green trajectories) in the inside part of the rotor. The trajectories in the external domain (in red) are not guided by the rotor corner. (d) A half rotor fixed in its corner rotates randomly with a mean angular velocity equal to zero; the scale bar is equal to $26 \mathrm{~mm}$. (e) A half rotor fixed at one of its extremities shows biased random rotation about its anchoring point; the scale bar is equal to $33 \mathrm{~mm}$. [In (d) and (e) the stroboscopic images of the half rotor, the motion is followed over $70 \mathrm{~s}$ at time intervals of $10 \mathrm{~s}$; the parameters are $L_{f}=4.4 \mathrm{~mm}$ and $U=2.8 \times 10^{-2} \mathrm{~m} \mathrm{~s}^{-1}$.]

of $U$ and the width $L_{f}$. Here $L_{R}$ is the distance between the pivot and the corner of the rotor. The function $f\left(T_{B}\right)$ is the coupling factor between the bundles and the rotor. For small rotors $\left(L_{r}<L_{f}\right)$ $f\left(T_{B}\right)=0$ [Fig. 2(f)]. For large rotors, $f\left(T_{B}\right)$ should reflect the bundle's finite lifetime. The simplest choice is $f\left(T_{B}\right) \sim T_{B}$, which leads to $f\left(T_{B}\right) \sim 1 / U$, in agreement with the observation of Fig. 3(c). Therefore, the model predicts that the mean angular velocity of a rotor should scale as $\left\langle V_{\theta}\right\rangle=P / C_{s} \sim U / L_{f}$. We indeed observe that the rotation velocity is proportional to the rms velocity $U$ of the horizontal fluid flow [Fig. 2(e)]. This simple model also predicts that the $Z$ rotor is more efficient at smaller characteristic scales of the flow $L_{f}$ [Fig. 2(f)]. We can also roughly estimate the efficiency rate $\eta$ of turbulent energy conversion of a rotor as $\eta=J\left\langle V_{\theta}\right\rangle^{2} / m_{f} U^{2}$, where $m_{f}=\rho h\left(2 L_{r}\right)^{2}$ is the mass of fluid interacting with the rotor, $\rho$ is the water density, and $h$ is the rotor height. Such estimation gives $\eta \approx 0.3$ for the rotor shown in Fig. 2(b).

The drag coefficient of an asymmetric object is a point that clearly would deserve further investigation. Indeed, one can argue that asymmetric objects might experience a direction-dependent drag force which would favor the directional motion observed. We have recently measured the 
propulsion force $F_{p}$ acting on the rotor by using optical fiber cantilevers [27]. In this type of measurement, the floating object is trapped by the optical fiber and this allows us to disentangle effects due to an anisotropic drag from those related to true propulsive force. The measurements confirmed that rotors or winged objects can actually extract the energy of the turbulent flow.

From a fundamental viewpoint, our results are in line with Curie's principle according to which the rectification of erratic motion is possible if temporal and spatial symmetries of the motion are broken [28]. In our experiments, time and spatial asymmetries arise from the finite flow memory and the rotor chirality. The emergence of directed motion from a chaotic environment [29] has been studied in a range of physical contexts in quantum systems [30], in microscale systems [31,32] where thermal noise plays an important role, and in the macroscopic world. Recently, this topic has seen two interesting experimental developments: complex ratchet effects discovered in a granular gas [33] and microscopic carriers powered by active baths [34] (a particular example of such an active bath is bacterial turbulence).

Beyond the conceptual analogies related to the possibility of rectification in an out-of-equilibrium system, it is interesting to discuss the differences between the ratchet mechanism at play in these two systems and ours. In a granular gas, chaos is generated by particle collisions and the ratchet effect occurs as a result of the inelasticity of those collisions. Microscopic carriers are based on out-of-equilibrium active baths which are dominated by viscous dissipation such that inertia effects are negligible (for instance, bacterial turbulence is observed for Reynolds number much smaller than 1 , typically $10^{-5}$ ). In this system, rectification of the bacteria motion occurs due to polar ordered bacteria trapped in the cusp of the carrier which is shielded from the outside turbulent fluctuations [35]. Hydrodynamic turbulence, on the other hand, is a chaotic state of a macroscopic system generated and dominated by inertia. The physical properties of hydrodynamic turbulence are obviously different from the recently discovered active bacterial turbulence [34,36]. The vehicles reported here are coupled to turbulent fluctuations on a fluid surface at typical Reynolds number of the order of 100; those hydrodynamical fluctuations have a complex spatiotemporal structure whose origin and features close to a wall are quite different from those of fluctuations observed in either bacterial turbulence or granular gases. From the fluid dynamicist point of view, the ability of bounded 2D turbulence to self-organize at large scale promises a versatile method for energy conversion. This study is just an initial step towards exploring how the properties of $2 \mathrm{D}$ turbulence in the vicinity of a moving wall can be used to harness its kinetic energy.

It is interesting to note that Couder and co-workers have discovered a different kind of propulsion mechanism occurring below the Faraday threshold instability on the surface of viscous liquids [37,38]. In these experiments, a millimeter-size droplet bouncing on the vibrated surface becomes a walker moving at constant speed when it couples to the localized surface wave, or pilot waves, it generates. Though it is observed in a similar setup, the operational principle of turbulence driven vehicle relies on totally different physics. Beyond the threshold instability, in weakly viscous liquid, steep Faraday waves generate vorticity on the liquid surface which fuels an inverse energy cascade. To create a propulsive coupling, the shape and size of a vehicle must be adapted to the characteristics of the underlying fabric of wave-driven turbulence [Fig. 2(f)].

In conclusion, the key to the extraction of useful work from 2D turbulent flows is the efficient coupling of an object to coherent structures present in the flow. In our experiments, coherent bundles transfer their momentum to the L-shaped arms of a floating object. Depending on the geometric configuration of these arms, a self-propelled vehicle or a rotor can be created.

\section{ACKNOWLEDGMENTS}

We thank Benjamin Faber for his contribution to developing the bundle detection technique. This work was supported by the Australian Research Council's Discovery Projects funding scheme (Grants No. DP150103468 and No. DP160100863). N.F. acknowledges support from the Australian Research Council's DECRA (Grant No. DE160100742). H.X. acknowledges support from the Australian Research Council's Future Fellowship (Grant No. FT140100067). 
[1] G. K. Batchelor, The Theory of Homogeneous Turbulence (Cambridge University Press, Cambridge, 1953).

[2] G. I. Taylor, Diffusion by continuous movements, Proc. London Math. Soc. s2-20, 196 (1921).

[3] D. J. C. MacKay, Sustainable Energy—Without the hot air (UIT Cambridge, Cambridge, 2009).

[4] M. Bourgoin, N. T. Ouellette, H. Xu, J. Berg, and E. Bodenschatz, The role of pair dispersion in turbulent flow, Science 311, 835 (2006).

[5] F. Toschi and E. Bodenschatz, Lagrangian properties of particles in turbulence, Annu. Rev. Fluid Mech. 41, 375 (2009).

[6] R. Kraichnan and D. Montgomery, Two-dimensional turbulence, Rep. Prog. Phys. 43, 547 (1980).

[7] Y. Couder, J. M. Chomaz, and M. Rabaud, On the hydrodynamics of soap films, Physica D 37, 384 (1989).

[8] G. Falkovich, G. Boffetta, M. Shats, and A. S. Lanotte, Introduction to Focus Issue: Two-dimensional turbulence, Phys. Fluids 29, 110901 (2017).

[9] H. Kellay, Hydrodynamics experiments with soap films and soap bubbles: A short review of recent experiments, Phys. Fluids 29, 111113 (2017).

[10] H. Xia and N. Francois, Two-dimensional turbulence in three-dimensional flows, Phys. Fluids 29, 111107 (2017).

[11] R. Kraichnan, Inertial ranges in two-dimensional turbulence, Phys. Fluids 10, 1417 (1967).

[12] J. Sommeria, Experimental study of two-dimensional inverse energy cascade in a square box, J. Fluid Mech. 170, 139 (1986).

[13] H. Xia, M. Shats, and G. Falkovich, Spectrally condensed turbulence in thin layers, Phys. Fluids 21, 125101 (2009).

[14] N. Francois, H. Xia, H. Punzmann, and M. Shats, Inverse Energy Cascade and Emergence of Large Coherent Vortices in Turbulence Driven by Faraday Waves, Phys. Rev. Lett. 110, 194501 (2013).

[15] A. V. Kameke, F. Huhn, G. Fernandez-Garcia, A. P. Munuzuri, and V. Perez-Munuzuri, Double Cascade Turbulence and Richardson Dispersion in a Horizontal Fluid Flow Induced by Faraday Waves, Phys. Rev. Lett. 107, 074502 (2011).

[16] N. Francois, H. Xia, H. Punzmann, S. Ramsden, and M. Shats, Three-Dimensional Fluid Motion in Faraday Waves: Creation of Vorticity and Generation of Two-Dimensional Turbulence, Phys. Rev. X 4, 021021 (2014).

[17] H. Xia, N. Francois, H. Punzmann, and M. Shats, Lagrangian scale of particle dispersion in turbulence, Nat. Commun. 4, 2013 (2013).

[18] N. Francois, H. Xia, H. Punzmann, B. Faber, and M. Shats, Braid entropy of two-dimensional turbulence, Sci. Rep. 5, 18564 (2015).

[19] See Supplemental Material at http://link.aps.org/supplemental/10.1103/PhysRevFluids.3.124602 for details on experimental methods, image analysis, flow characterization and a video of the motion of a beam and a rotor placed in wave driven turbulence.

[20] N. Francois, H. Xia, H. Punzmann, P. Fontana, and M. Shats, Wave-based liquid-interface metamaterials, Nat. Commun. 8, 14325 (2017).

[21] H. Punzmann, N. Francois, H. Xia, G. Falkovich, and M. Shats, Generation and reversal of surface flows by propagating waves, Nat. Phys. 10, 658 (2014).

[22] N. Francois, H. Xia, H. Punzmann, and M. Shats, Wave-particle interaction in the Faraday waves, Eur. Phys. J. E 38, 106 (2015).

[23] A. E. Hansen, E. Schröder, P. Alstrøm, J. S. Andersen, and M. T. Levinsen, Fractal Particle Trajectories in Capillary Waves: Imprint of Wavelength, Phys. Rev. Lett. 79, 1845 (1997).

[24] N. Francois, H. Xia, H. Punzmann, T. Combriat, and M. Shats, Inhibition of wave driven two-dimensional turbulence by viscoelastic films of proteins, Phys. Rev. E 92, 023027 (2015).

[25] K. J. Welch, A. Liebman-Pelaez, and E. I. Corwin, Fluids by design using chaotic surface waves to create a metafluid that is Newtonian, thermal, and entirely tunable, Proc. Natl Acad. Sci. USA 113, 10807 (2016).

[26] M. Allshouse and J. L. Thiffeault, Detecting coherent structures using braids, Physica D 241, 95 (2012).

[27] N. Francois, D. Lasne, Y. Amarouchene, B. Lounis, and H. Kellay, Drag Enhancement with Polymers, Phys. Rev. Lett. 100, 018302 (2008). 
[28] P. Curie, Sur la symétrie dans les phenomènes physiques, symétrie d'un champ électrique et d'un champ magnétique, J. Phys. (Paris) 3, 393 (1894).

[29] R. P. Feynman, R. B. Leighton, and M. Sands, The Feynman Lectures on Physics (Addison-Wesley, Reading, 1963), Vol. 1, Chap. 46.

[30] T. Emig, Casimir-Force-Driven Ratchets, Phys. Rev. Lett. 98, 160801 (2007).

[31] P. Reimann, Brownian motors: Noisy transport far from equilibrium, Phys. Rep. 361, 57 (2002).

[32] J. Rousselet, L. Salome, A. Adjari, and J. Prost, Directional motion of Brownian particles induced by a periodic asymmetric potential, Nature (London) 370, 446 (1994).

[33] P. Eshuis, K. van der Weele, D. Lohse, and D. van der Meer, Experimental Realization of a Rotational Ratchet in a Granular Gas, Phys. Rev. Lett. 104, 248001 (2010).

[34] C. Bechinger, R. Di Leonardo, H. Lowen, C. Reichhardt, G. Volpe, and G. Volpe, Active particles in complex and crowded environments, Rev. Mod. Phys. 88, 045006 (2016).

[35] A. Kaiser, A. Peshkov, A. Sokolov, B. ten Hagen, H. Löwden, and I. S. Aranson, Transport Powered by Bacterial Turbulence, Phys. Rev. Lett. 112, 158101 (2014).

[36] I. Aranson, Viewpoint: The aquatic dance of bacteria, Physics 6, 61 (2013).

[37] Y. Couder, S. Protiere, E. Fort, and A. Boudaoud, Dynamical phenomena: Walking and orbiting droplets, Nature (London) 437, 208 (2005).

[38] S. Protiere, A. Boudaoud, and Y. Couder, Particle-wave association on a fluid interface, J. Fluid Mech. 554, 85 (2006). 